\title{
Coronavirus Disease 2019 - The Principles of the Curve, Explained Simply
}

\author{
Yelda Jozaghi, MD, FRCSC ${ }^{1}$ \\ ${ }^{1}$ The University of Texas MD Anderson Cancer Center
}

May 11, 2020

\begin{abstract}
Much has been discussed about the curve of COVID-19 as it ravages through our countries, our cities, our homes. Politicians readily refer to it in addressing the various nations of the world. They ask us to "flatten the curve to save lives" 1,2 . Effectively, the way to flatten the curve is to abide by social distancing measures.

Nevertheless, even among medical specialists, there are common misconception about the curve and how it affects population outcomes. Understanding the fundamental characteristics of a problem can allow us to see the problem with more clarity. Herein, I aim to provide a simple understanding of the various population dynamics at play. We will review how principles such as the area under the curve and thethreshold of capacity can be conceptualized.

The discussion will begin as rudimentary. However, it will add gentle layers of complexity. Hopefully, by the end, it will provide the reader a sense of insight.
\end{abstract}

Yelda Jozaghi, MD, FRCSC

Department of Head and Neck Surgery

The University of Texas MD Anderson Cancer Center

Houston, TX, USA.

\begin{abstract}
Much has been discussed about the curve of COVID-19 as it ravages through our countries, our cities, our homes. Politicians readily refer to it in addressing the various nations of the world. They ask us to "flatten the curve to save lives" 1,2 . Effectively, the way to flatten the curve is to abide by social distancing measures.

Nevertheless, even among medical specialists, there are common misconception about the curve and how it affects population outcomes. Understanding the fundamental characteristics of a problem can allow us to see the problem with more clarity. Herein, I aim to provide a simple understanding of the various population dynamics at play. We will review how principles such as the area under the curve and thethreshold of capacity can be conceptualized.

The discussion will begin as rudimentary. However, it will add gentle layers of complexity. Hopefully, by the end, it will provide the reader a sense of insight.
\end{abstract}

Defining the Curve

What are it axes? 
The $\mathrm{X}$ axis is a measure of time.

The $\mathrm{Y}$ axis represents the number of people affected during that time (the rate), may it be by infection, mortality, or recovery depending on the type of curve.

\section{"The Curve", is there only one?}

Though this concept of "The Curve" is often referred to as a single entity, there are actually various curves a play. In a simple way to conceptualize the population dynamics, we can define 3 fundamental curves: the infection curve, the mortality curve, and the recovery curve.

The infection curve describes the rates of COVID-19 infection across our society. [graph 1].

[Insert graph 1]

The mortality curve describes the mortality rates across society. The peak of this curve is offset to the right on the $\mathrm{X}$-axis as compared to the infection curve [graph 2]. The peak to peak delta $\left(\mathrm{X}_{\mathrm{PM}^{-}} \mathrm{X}_{\mathrm{PI}}\right)$ will correspond to the average time duration between infection diagnosis and mortality.

[Insert graph 2]

The recovery curve describes the recovery rates across society. The peak of this curve is offset to the right on the $\mathrm{X}$-axis as compared to the infection curve [graph 3]. The peak to peak time difference $(\Delta \mathrm{X})$ will correspond to the average time duration between infection diagnosis and recovery. The corresponding peak to peak population ratio delta-Y will represent the number of people who passed away at the peak. The peak to peak ratio ( $\mathrm{Y}_{\text {peak-recovery }} / \mathrm{Y}_{\text {peak-infection }}$ ) will represent the recovery rate at the peak of disease [graph 3].

[Insert graph 3]

\section{Area under the curve, what does it represent?}

Without discussing the calculus of these functions, understanding the "area under the curve" is integral to conceptualizing these curves. The 2-dimensional shaded area below the curve will represent the total number of cases. We will designate this area as AUC. Depending on the type of curve, it will represent the total number of measured infections, mortalities, and recoveries. Hence the following formula will apply:

$\mathrm{AUC}$ infection $=$ AUC mortality + AUC recovery.

In other words, the total number of people infected will be equal to the number of people who pass away added to the number of people who recover.

[Insert graph 4]

\section{How external factors save lives}

Flattening the curve does not necessarily save lives on its own

To save lives, you must decrease the area under the Mortality Curve. Flattening the curve will inherently modify the rates of infection, mortality, and recovery. By flattening the curve with social distancing, the shape of the curve is different, the slopes of the tangents are different. However, the area under the curve does not necessarily change. Flattening the curve, can bring down the peak andwiden the base, maintaining the same $A U C$ [graph 5].

Dr Robert Katz, founding director of the Yale-Griffin Prevention Research Center, has been quoted on this topic: "If all we do is flatten the curve, you don't prevent deaths, you just change the dates" 3 .

This is why. To save lives, you must decrease the area under the curve. Flattening the curve, does not necessarily achieve that on its own. It has the potential to save lives when combined with the following factors.

[Insert graph 5] 


\section{Avoiding the threshold of hospital capacity. We can't all get sick at once.}

Avoiding the threshold of hospital capacity is one way by which "flattening the curve" can save lives ${ }^{1,4}$. By threshold of capacity, I am referring the maximal number of people the healthcare system can accommodate. Once we reach that limit, mortality rises, not because we couldn't have saved those lives. It rises because we don't have the resources to save them [graph 6].

Basically, we can't all get sick at the same time. That's because there aren't enough ICU beds or ventilators. [Insert graph 6]

\section{A potential cure. Buying time until a vaccine or remedy.}

Flattening the curve can also potentially save lives because it buys us time until we find a cure, preventive vaccine, or effective remedy.

If a cure is found, then the AUC at the tail end of the flattened infection curve will be larger than that of a non-flattened curve. Those are effectively less people infected (on the infection curve), and lives saved (on the mortality curve).

[Insert graph 7]

The AUC after the cure represents the number of people who avoided the illness. That number is larger on a flattened curve. Ergo, flattening the curve can save more people from being infected if a cure is found.

\section{How does this translate to a strategy? 3 tiers}

Putting all this together, there are 3 fundamental interventions governments can enact to decrease the mortality.

Strategy 1. Flatten the curve, bring down the peakSocial distancing measures will bring down the peak of the curve.

[Insert graph 8]

Strategy 2. Raise the threshold of hospital capacity. This consists of building more ventilators, increasing the number of ICU beds, ICU personnel, emergency room, and medical ward capacity. Basically, the aim is to raise the threshold of hospital capacity so high, that the curve doesn't cross it.

[Insert graph 9]

Strategy 3. Invest in a cure or treatment.An earlier cure in combination with a flattened curve will maximize the number of lives saved.

[Insert graph 10]

To be clear, such strategies are designed strictly to minimizing mortality across society during the pandemic. This article does not discuss strategies on returning to normalcy. Nevertheless, the ideal trigger to transition from one strategy to the other would be the development of a cure such as a vaccine or effective treatment.

However, if this cannot be found in time, our only option may be to gradually return to life on the downslope of the curve. Doing so, hoping that herd immunity may provide protection to the vulnerable. In such a scenario, mathematical modeling could to inform us on which part of the curve would optimize herd immunity and minimize a rebound.

\section{References}

1. Anderson RM, Heesterbeek H, Klinkenberg D, Hollingsworth TD. How will country-based mitigation measures influence the course of the COVID-19 epidemic? Lancet 2020; 395 (10228): 931-4.

2. Tanne JH, Hayasaki E, Zastrow M, Pulla P, Smith P, Rada AG. Covid-19: how doctors and healthcare systems are tackling coronavirus worldwide. BMJ 2020; 368. 
3. Katz DL. Real Time with Bill Maher, S18 E12, April 24, 2020. https://davidkatzmd.com/coronavirusinformation-and-resources/. Visited April 27, 2020. Published April 24, 2020.

4. Zhang Z, Yao W, Wang Y, Long C, Fu X. Wuhan and Hubei COVID-19 mortality analysis reveals the critical role of timely supply of medical resources. J Infect 2020 Mar 21; S0163-4453(20)30145-6.
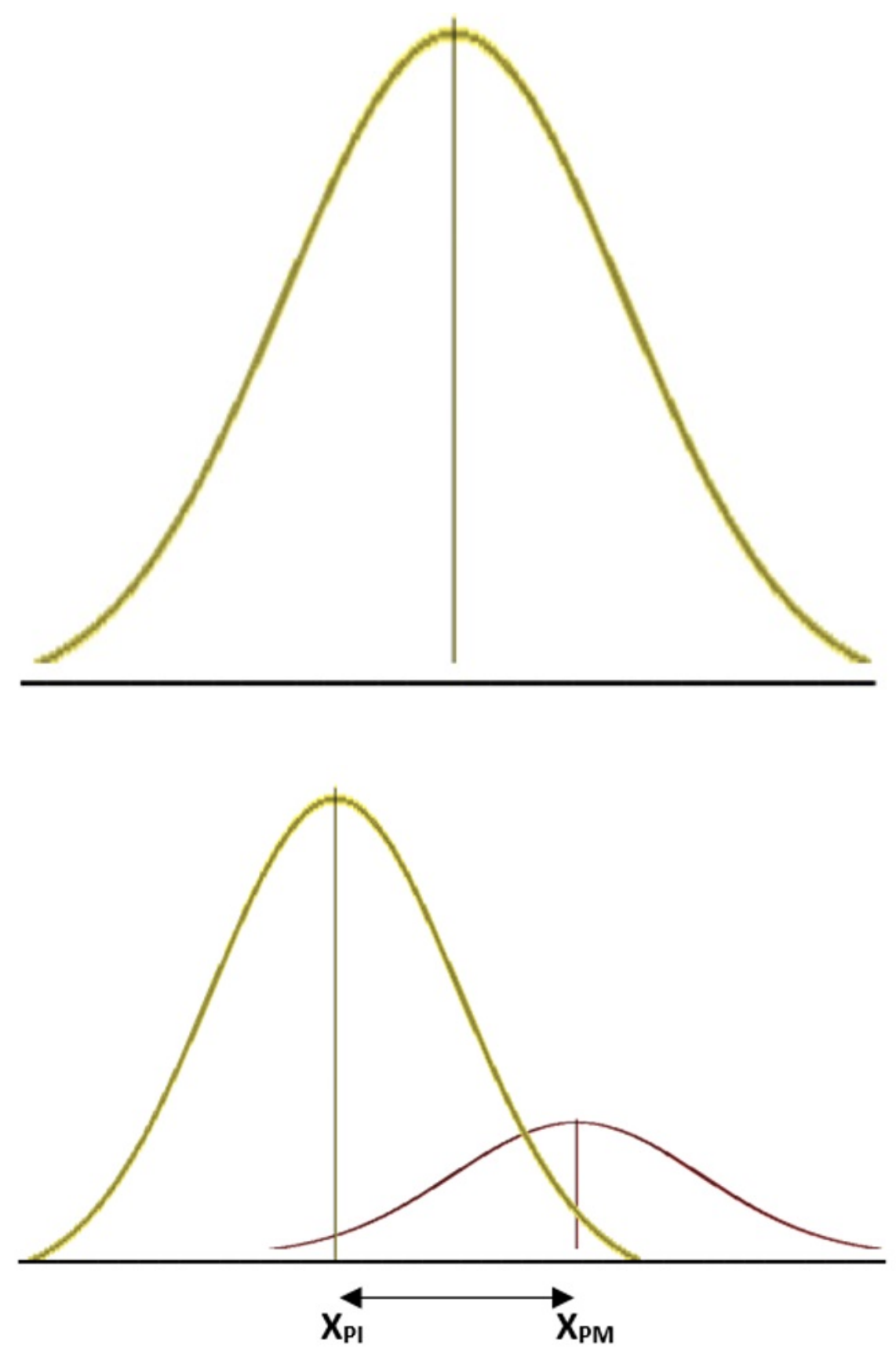


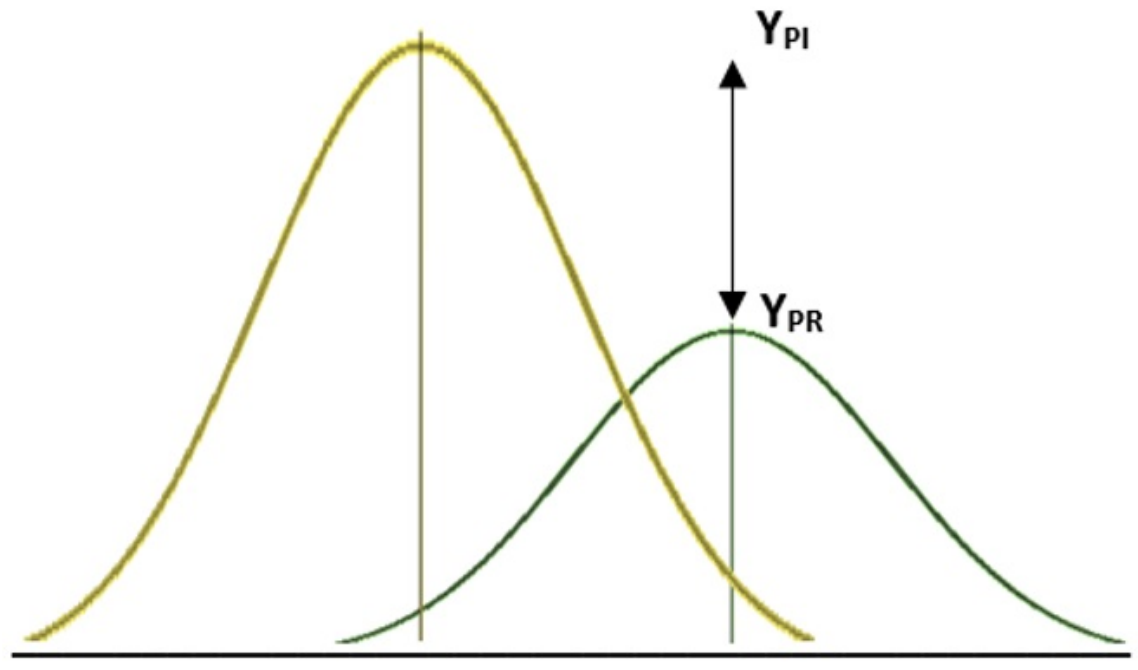

AUC infection - AUC recovery = AUC mortality
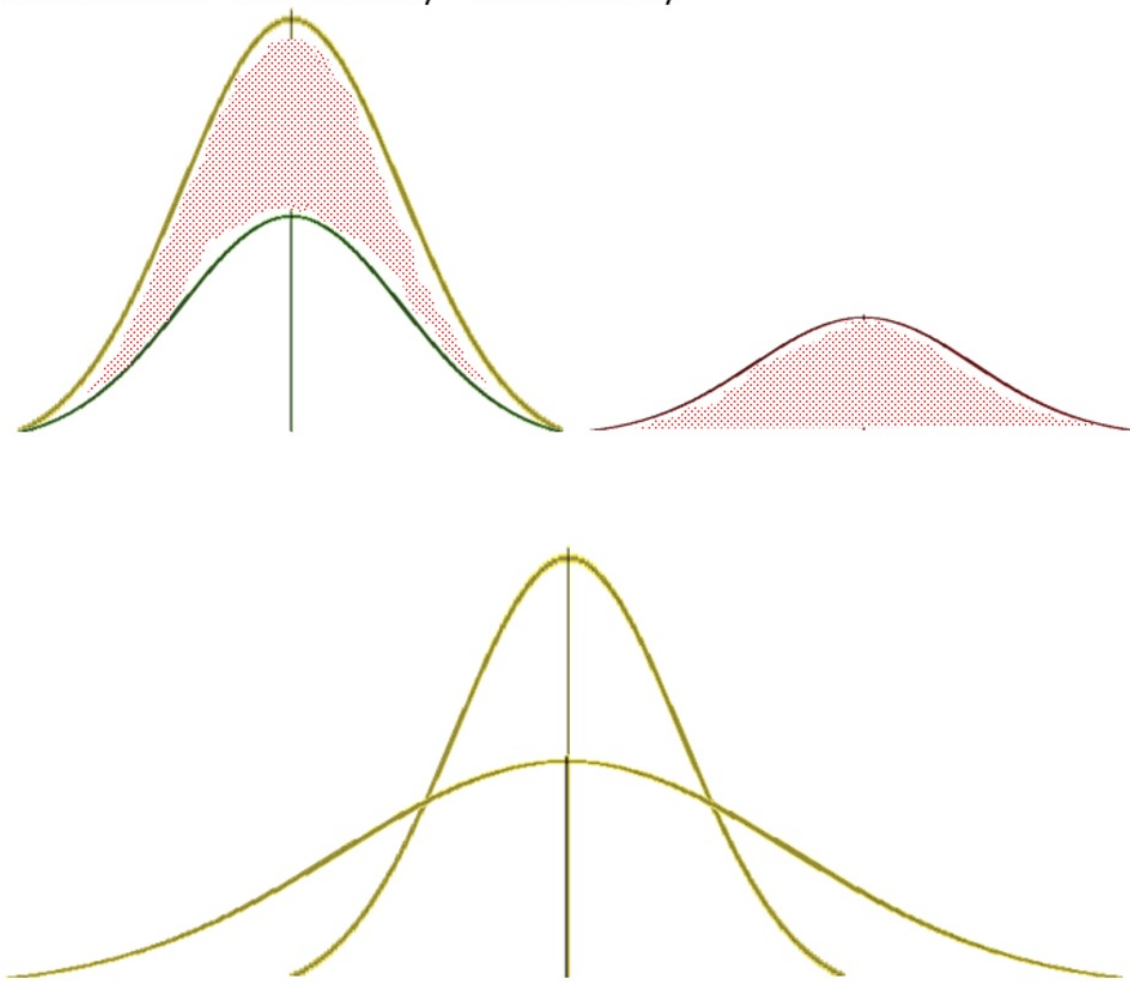

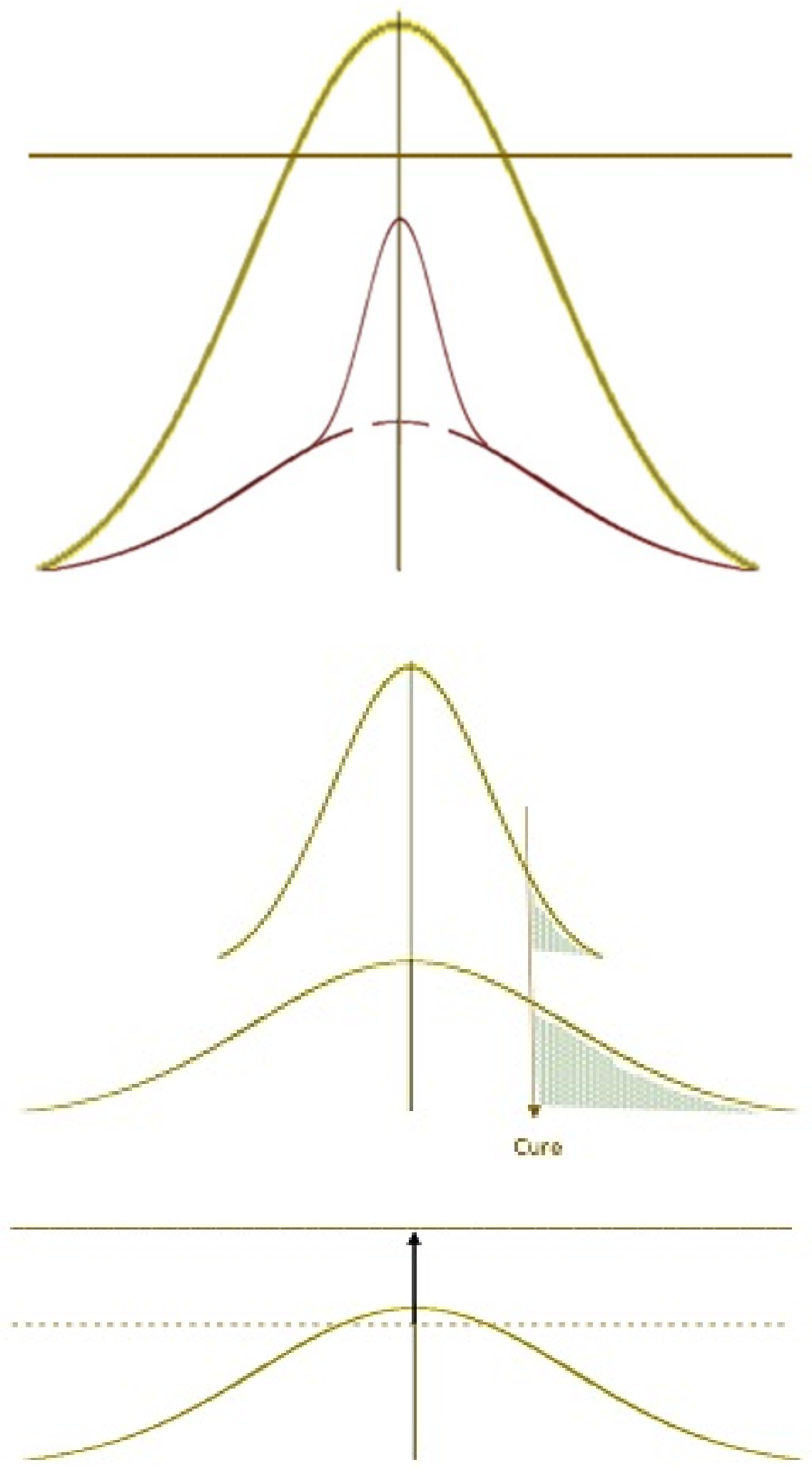

6 

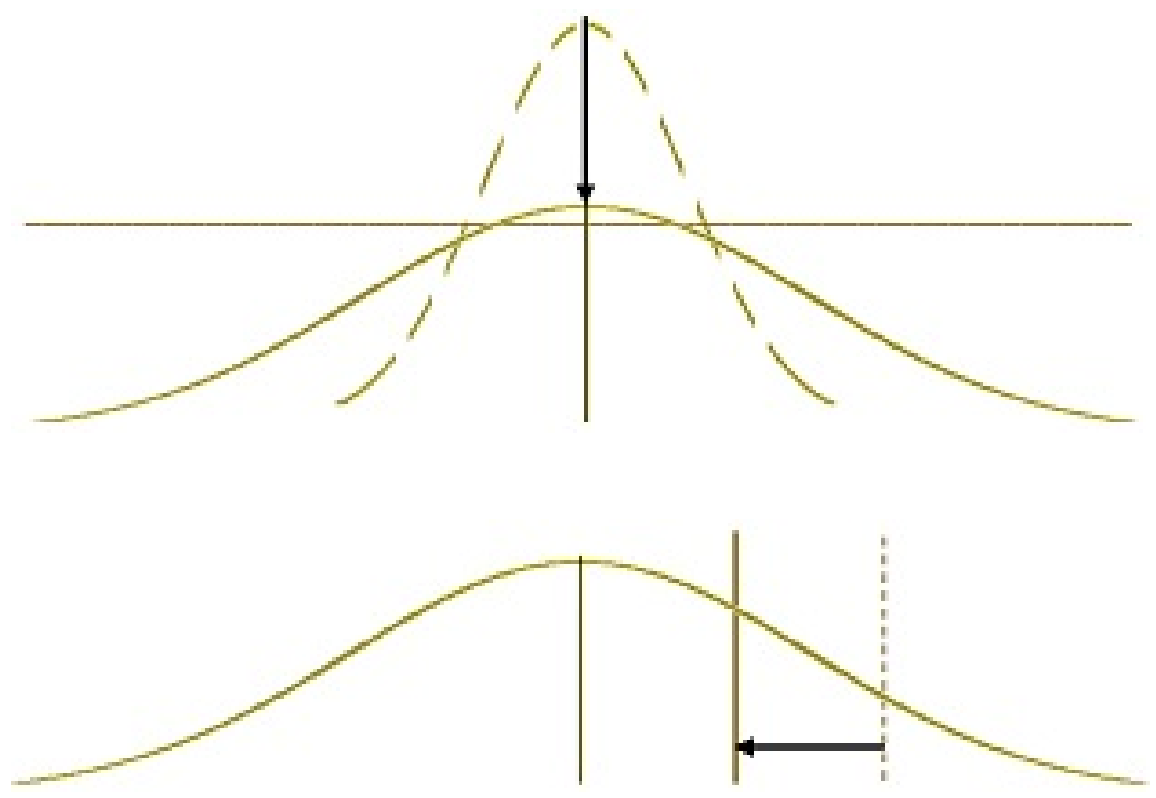

7 